\title{
Oriented Pattern Analysis for Streak Detection in Dermoscopy Images
}

\author{
Maryam Sadeghi ${ }^{1,2,3}$, Tim K. Lee ${ }^{1,2,3}$, David McLean ${ }^{2}$, \\ Harvey Lui ${ }^{2}$, and M. Stella Atkins ${ }^{1,2}$ \\ 1 School of Computing Science, Simon Fraser University, Canada \\ msa68@sfu.ca \\ 2 Department of Dermatology and Skin Science, University of British Columbia, Canada \\ ${ }^{3}$ Cancer Control Research Program, BC Cancer Research Center, Canada
}

\begin{abstract}
There is an increasing demand for automated detection and analysis of dermoscopy structures and malignancy clues such as streaks in dermoscopy images, for computer-aided early diagnosis of deadly melanoma. This paper presents a novel approach for streak detection and visualization on dermoscopic images. We tackle the detection of streaks by means of ridge and valley estimation. Orientation estimation and correction is applied to detect low contrast and fuzzy streaks lines, and candidate streaks are used to classify dermoscopy images into streaks Absent or Present with the AUC of $90.5 \%$ on 300 dermoscopy images. Our approach can also detect starburst pattern of regular streaks using detected linear structures with accuracy of $81.5 \%$ and AUC of $87.7 \%$.
\end{abstract}

\section{Introduction}

Melanoma is the most deadly form of skin cancer, yet treatable via excision if detected early. There is, therefore, a demand to develop computer-aided diagnostic systems to facilitate the early detection of melanoma. Dermoscopy, also known as epiluminescence microscopy (ELM), is an in-vivo noninvasive skin imaging method useful for the early recognition of malignant melanoma. For melanoma detection, in almost all of the dermoscopy methods, evaluation of dermoscopic structures focuses on structural features such as pigment network, streaks, dots, and globules [1].

Clinical Definition: Streaks is a term used interchangeably with radial streaming or pseudopods. Radial streaming is a linear extension of pigment at the periphery of a lesion radially arranged linear structures in the growth direction, and pseudopods represent finger-like projections of dark pigment (brown to black) at the periphery of the lesion. In order to ensure accurate recognition, streaks are numerated only when at least 3 near linear and parallel structures are clearly visible [1]. Streaks are local dermoscopy features of skin lesions, however they can correlate with a global pattern of skin lesions called a starburst pattern if symmetrically arranged over the entire lesion.

Mathematical Definition: The above clinical definition is translated to mathematical concepts with justified parameters to be captured by image processing techniques: 1) Streaks are 3 or more linear structures co-radially oriented in the boundary which is a contour with the thickness equal to $1 / 3$ of the minor axis of the lesion. 2) Streaks are 


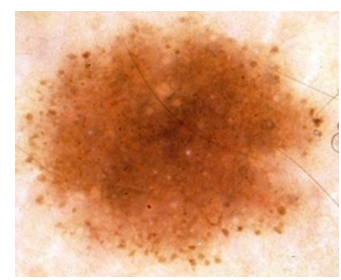

(a)

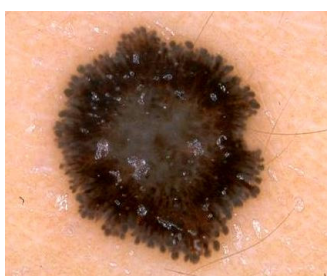

(b)

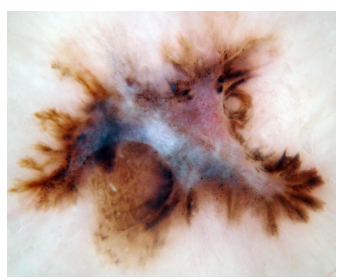

(c)

Fig. 1. Examples of absent, regular and irregular streaks. (a) shows a lesion without streaks ( $A b$ sent). (b) illustrates a lesion with a complete symmetric regular streaks pattern called starburst (Present), and in (c), a melanoma lesion with irregular streaks is shown (Present).

darker than their neighborhood. 3) Streaks are shorter than the $1 / 3$ of the minor axis of the lesion and they should be longer than one percent of the major axis. 4) Streaks do not branch and their curvature is smaller than one.

Figure 1 shows examples of lesions with no streaks (Absent), regular (Present), and irregular (Present) streaks. Figure 1 -a shows Absent, 1 - $b$ shows a starburst pattern, and Figure 1-c shows a lesion with irregular streaks and partial pattern.

Diagnostic Importance: Streaks are important morphologic expressions of malignant melanoma, specifically melanoma in the radial growth phase [2]. Irregular streaks is one of the most critical features (included in almost all of dermoscopy procedures) that shows the high association with melanoma. Also Menzies et al. [2] found pseudopods to be one of the most specific features of superficial spreading melanoma which is a subset of malignant melanoma. In addition, symmetric streaks (starburst pattern) is one of the specific dermoscopic criteria to differentiate usually benign Spitz nevi (a dark nevus common in children) from melanoma, thus increasing diagnostic accuracy for pigmented Spitz nevus. However, all lesions in adults exhibiting a starburst pattern should be excised for histopathological evaluation [2]. Therefore detection of streaks can be a significant step towards computer-aided diagnosis of skin lesions and melanoma detection.

As a fundamental step towards computer-aided diagnosis of skin cancers, automatic detection of many of these dermoscopic structures have been recently addressed in the literature [34]. However, the automatic detection of streaks has only recently been investigated [5]6]. Streaks on dermoscopy images usually are difficult to detect since they are not perfect linear structures, but often fuzzy and low-contrast oriented intensities. Furthermore, streaks may have unpredictable spatial distribution (partial pattern) with just a few streaks lines in a small region of a lesion. Therefore, it is not easy to detect them using general oriented pattern analysis. Mirzaalian et al. [6] have used a machine-learning approach for classifying streaks in dermoscopic images. Although the methodology is interesting, it, unfortunately, has been tested on a small number (99) of dermoscopic images with wide exclusion criteria. It is not clear how the method generalizes to all conditions of dermoscopic images captured in a dermatologist clinic.

This paper presents a novel method to estimate, enhance, detect and visualize streaks. 


\section{Method}

After a pre-processing step, multi-scale Laplacian of Gaussian is applied to detect dermoscopy structures with Gaussian cross-sectional profile. After finding linear structures, the orientation flow of the image is analyzed to determine the orientation of detected objects in the orientation flow to select linear structures of candidate streaks. Finally, chromatic and textural features of detected line segments are used to classify the lesions into Absent or Present images. These steps are shown in Figure 2.

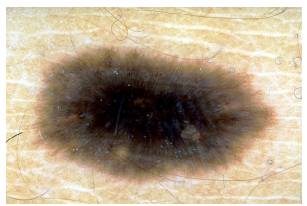

(a)

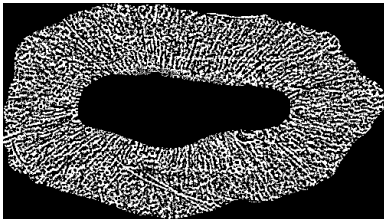

(d)

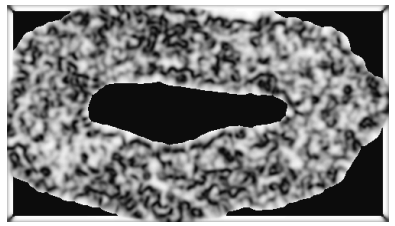

(g)

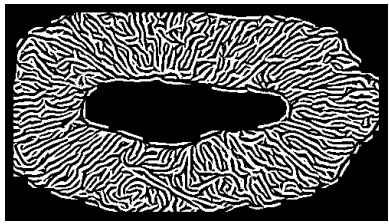

(j)

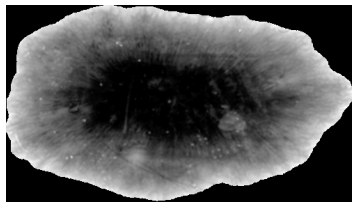

(b)

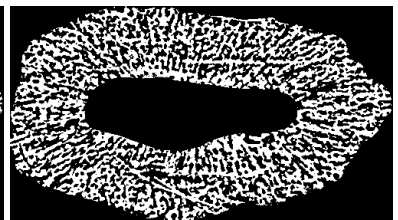

(e)

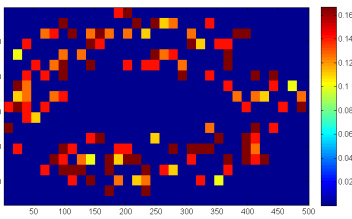

(h)

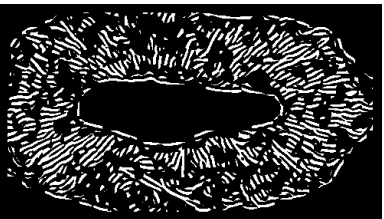

(k)

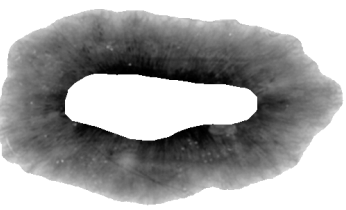

(c)

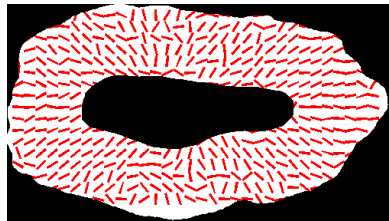

(f)

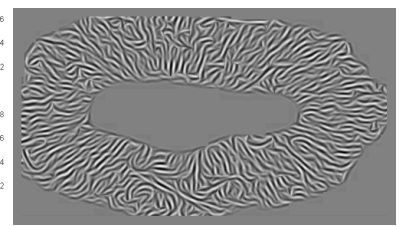

(i)

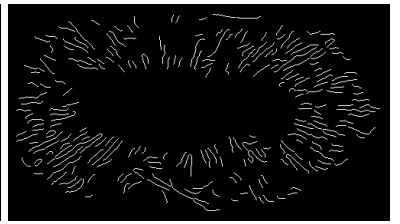

(1)

Fig. 2. The overview of the method. (a) shows a lesion with regular streaks and (b) illustrates the result of segmentation re-orientated and sharpening in the $\mathrm{L}^{*}$ channel. (c) illustrates the region of interest that will be processed to find streaks. (d), and (e) show the LOG filter responses in the two scales $h$ size $_{k}=3,9$ respectively. In (f), the directional flow is plotted with red lines for $h_{\text {size }}=9$, and $(\mathrm{g})$ shows the reliability of estimation. The median frequency of the parallel pattern, illustrated in (h), is used in Gabor filters applied to enhance the estimated orientation shown in (i). (j) shows the binary image of the enhanced orientation with 1 for ridges and 0 for valleys. $(\mathrm{k})$ is created from $(\mathrm{g})$ and $(\mathrm{j})$ by removing pixels with reliability $\leq 50 \%$ and skeleton of the result is shown in (l) as detected candidate streaks structures. 


\subsection{Preprocessing}

First the lesion is segmented using Wighton et al.'s method [7] and the orientation of the lesion is found, and the image is rotated to align the major axis horizontally since the major axis represents the lesion growth direction. Then, to have a relatively uniform image size, the lesion is re-sized so that its major axis occupies 500 pixels. Finally, the image is enhanced using a simple $3 \times 3$ high pass filter that removes the low frequency noise [3]. To get a single plane luminance image, the given RGB image is converted to the $\mathrm{L}^{*} \mathrm{a} * \mathrm{~b}$ color-space and $\mathrm{L}^{*}$, is used for the rest of our analysis (Figure $2-\mathrm{b}$ ).

\subsection{Identifying Linear Structures after Enhancing Orientation}

The region of interest (the boundary of the lesion) is found by computing the distance transform of the lesion mask. $1 / 3$ of the length of the lesion's minor-axis is used to compute the boundary area. Figure $2-\mathrm{c}$ shows the region of interest to find streaks.

Blob Detection Using LOG: Since streaks are linear structures with Gaussian crosssectional profiles, we detect them using Laplacian of Gaussian (LOG). To capture objects of different sizes a multi-scale approach is necessary. Thus, an input image $f(x, y)$ is filtered by rotationally symmetric LOG filters of size hsize $=3,5,7,9$ with a small value of 0.1 assigned to the standard deviation in order to achieve high sensitivity even to a small changes in intensity. At the end, we will union results of the four scales to form a multi-scale result. Figure 2-d and 2 -e show the LOG responses at two different scales of $h s i z e_{k}=3,9$ respectively.

Estimating Orientation: After finding linear structures by LOG, the orientation estimation is performed using the Averaged Squared Gradient Flow (ASGF) algorithm [8]. The reason for using squared gradient instead of the elementary gradient is that after computing the local orientation for a pixel, the estimation will be averaged over a block of $16^{*} 16$. Since a ridge line has two edges, the gradient vectors at both sides of a ridge are opposite to each other. Therefore, gradients cannot directly be averaged since opposite vectors will cancel each other, although they indicate the same ridge-valley orientation. Therefore, by applying ASGF that doubles the angles of the gradient vectors before averaging, opposite gradient vectors will point in the same direction and will reinforce each other. Also, the length of the gradient vectors is squared, as if the gradient vectors are considered as complex numbers that are squared. Thus, strong orientations have a higher vote in the average orientation than weaker orientations.

The qualitative analysis that was given above is made quantitative here. The algorithm starts by computing the gradients $G_{x}(i, j)$ and $G_{y}(i, j)$ at each pixel $(i, j)$ in image $I$. For doubling the angle and squaring the length in ASGF, the gradient vector is converted to polar coordinates, in which it is given by $\left[G_{\rho}, G_{\theta}\right]$ : 


$$
\begin{aligned}
G_{\rho} & =\sqrt{G_{y}^{2}+G_{x}^{2}}, \quad G_{\theta}=\tan ^{-1}\left(G_{y}, G_{x}\right) \\
G_{x} & =G_{\rho} * \cos \left(G_{\theta}\right), \quad G_{y}=G_{\rho} * \sin \left(G_{\theta}\right) \\
{\left[\begin{array}{c}
G_{s, x} \\
G_{s, y}
\end{array}\right] } & =\left[\begin{array}{c}
G_{\rho}{ }^{2} \cos \left(2 G_{\theta}\right) \\
G_{\rho}^{2} \sin \left(2 G_{\theta}\right)
\end{array}\right]=\left[\begin{array}{c}
G_{\rho}^{2}\left(\cos ^{2}\left(G_{\theta}\right)-\sin ^{2}\left(G_{\theta}\right)\right) \\
G_{\rho}^{2}\left(2 \sin \left(G_{\theta}\right) \cos \left(G_{\theta}\right)\right)
\end{array}\right]=\left[\begin{array}{c}
G_{x}^{2}-G_{y}^{2} \\
2 G_{x} G_{y}
\end{array}\right] \\
D F & =\frac{1}{2} \tan ^{-1}\left[\frac{2 G_{x} G_{y}}{G_{x}^{2}-G_{y}^{2}}\right]
\end{aligned}
$$

where $\left[\begin{array}{l}G_{s, x} \\ G_{s, y}\end{array}\right]$ is the squared gradient and DF is the directional flow of image $I$.

The image is divided into blocks of size $\mathrm{W}=16$. For each block, the local orientation centered at pixel $(i, j)$ is estimated and averaged using DF as follows:

$$
\left[\overline{G_{s, x}}\right]=\frac{1}{|W|}\left[\begin{array}{c}
\sum_{W} G_{x}^{2}-G_{y}^{2} \\
\sum_{W} 2 G_{x} G_{y}
\end{array}\right]
$$

To reduce the effect of noise on the estimated orientation, a low-pass filter (Gaussian) is used to modify the local ridge orientation. In order to apply the Gaussian filter, the orientation image is converted back to a continuous vector as follows:

$$
\begin{aligned}
& \Phi_{x}(i, j)=\cos \left(2 G_{\theta}(i, j)\right), \quad \Phi_{y}(i, j)=\sin \left(2 G_{\theta}(i, j)\right) \\
& \Phi_{x}^{\prime}(i, j)=\sum_{W} F(i, j) \Phi_{x}(i, j), \quad \Phi_{y}^{\prime}(i, j)=\sum_{W} F(i, j) \Phi_{y}(i, j)
\end{aligned}
$$

where $F$ is the Gaussian filter with unit integral and specified size $w_{\Phi}=5$. Now, the local orientation and its reliability (the coherence of the squared gradients given by [8]) can be computed at pixel $(i, j)$ using the following equation:

$$
O(i, j)=\frac{1}{2} \tan ^{-1}\left(\frac{\Phi_{y}^{\prime}(i, j)}{\Phi_{x}^{\prime}(i, j)}\right), \quad \text { Reliability }=\frac{\left|\sum_{W}\left(G_{s, x}, G_{s, y}\right)\right|}{\sum_{W}\left|\left(G_{s, x}, G_{s, y}\right)\right|} .
$$

which means if all squared gradient vectors are pointing in exactly the same direction, the sum of the vectors equals the modulus of the sum of the vectors, resulting in a coherence value of 1 . On the other hand, if the gradient vectors are random in all directions, the sum of them will be 0 , resulting in a coherence equal to 0 . This algorithm results in a smooth intensity flow orientation over the image (shown in Figure 2-f), and Figure $2 \mathrm{~g}$ shows the reliability map of the orientation estimation in the example image.

Estimating Ridge Frequency: After finding the local orientation and averaging for image blocks, the local ridge frequency is estimated by rotating the block so that the ridges are vertical. Then, the columns are projected down to find peaks. The frequency of ridges can be calculated by dividing the distance between the first and last peaks by (number of peaks -1 ), and finally the median frequency is computed over all the blocks in the image. Figure 3 a shows an example block the image given in Figure 3 -a. The result of rotating the block with the average block orientation to make it vertical is 


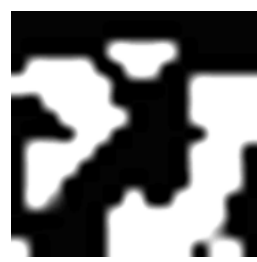

(a)

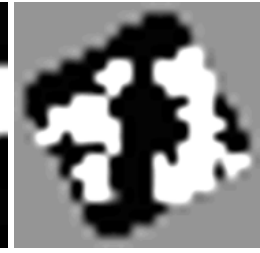

(b)

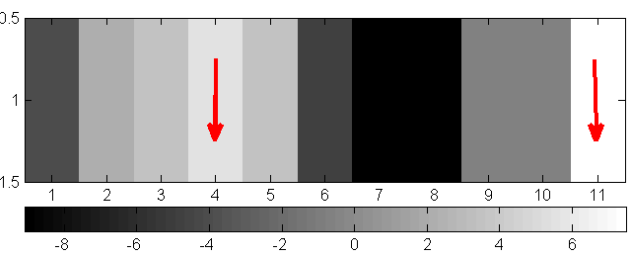

(c)

Fig. 3. Ridge frequency estimation. (a) shows an example block magnified for illustration (b) illustrates the result of rotation with average block orientation which is 30 degree for this block. (c) shows the projection result with red arrows pointing the peaks. The wavelength of this block is $11-4=7$ that results in frequency of $1 / 7=0.14$.

shown in Figure 3-b, and the projection step is shown in 3-c. Now peaks of the block can be easily found using the histogram of projection. The frequency can be found using these peaks and their wavelength. The final result of frequency estimation for all blocks of our example image (2)-d) is shown in Figure 2 -h.

Enhancing Orientation Image: From [9], a Gabor filter with tuned ridge frequency and orientation, can remove the noise efficiently while preserving true ridges and valleys. The even-symmetric Gabor filter has the general form of $g(x, y ; f, \theta, \sigma)=$ $\exp \left(-\frac{x^{\prime 2}+y^{\prime 2}}{2 \sigma^{2}}\right) \cos \left(2 \pi f x^{\prime}\right), x^{\prime}=x \cos \theta+y \sin \theta$, and $y^{\prime}=-x \sin \theta+y \cos \theta$, where $\sigma$ is the sigma of the Gaussian kernel in the filter, and $\mathrm{f}$ and $\theta$ are the corresponding median ridge frequency over the image and local orientation respectively. The result of this step is shown in Figure 2-i. Figure 2-j shows the binary image of the enhanced orientation created by thresholding ( 1 for ridges and 0 for valleys), and Figure 2. $\mathrm{k}$ is created from ( $\mathrm{j}$ ) and ( $\mathrm{g}$ ) by removing pixels with reliability $\leq 50 \%$. The skeleton of the result is shown in (1) as detected linear structures in the image. These line segments will be used for feature extraction in the next step.

Figure 4 demonstrates our streak detection method qualitatively. Figures $4 \mathrm{Ha}$, and 4-c illustrate absent images and their results are shown in 4-b and 4-d. In the second row, Figure 4. - and 4.g, shows two starburst lesions (regular streaks) with their streaks detected in Figure 4f $\mathrm{f}$ and 4 th respectively. Two melanomas with irregular streaks are shown in the third row with their corresponding results.

\subsection{Feature Extraction and Classification}

Based on our mathematical definitions of streaks, we propose a new set of 12 features called STR which includes three structural, six chromatic, and 3 textural characteristics of candidate streaks. In $S T R$, Structural set includes the number of candidate streaks in the image, average number of pixels of candidate streaks, and the ratio of the streaks size to the lesion size in pixels; Chromatic set consists of the mean, standard deviation and reciprocal of coefficient of variation (mean/stdev) of candidate streaks in $\mathrm{L}^{*}$ and $\mathrm{S}$, and std of $\mathrm{H}$; and Textural features are energy, contrast, and homogeneity of candidate streaks. We have also used common color and texture features [4] of the lesion itself 


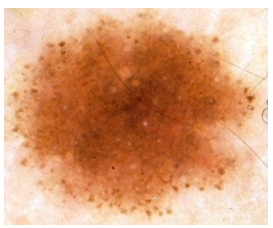

(a)

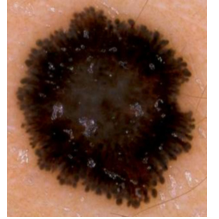

(e)

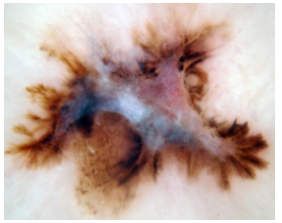

(i)

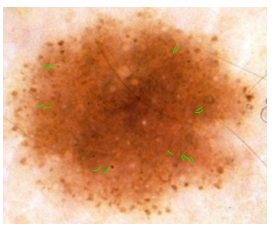

(b)

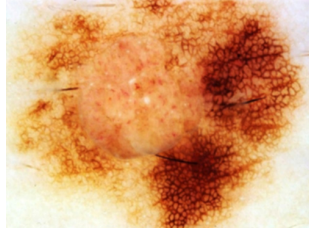

(c)

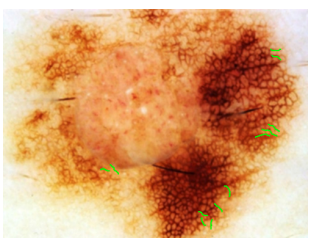

(d)

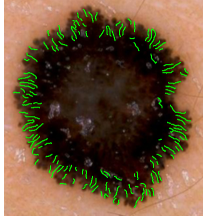

(f)

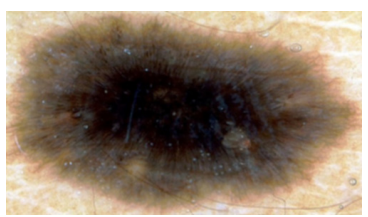

(g)

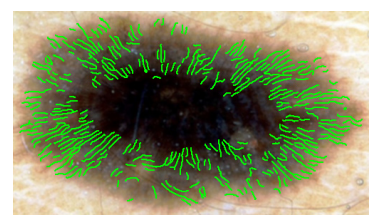

(h)

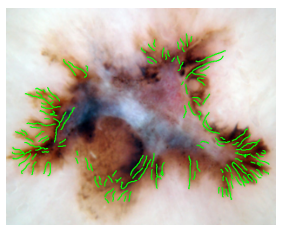

(j)

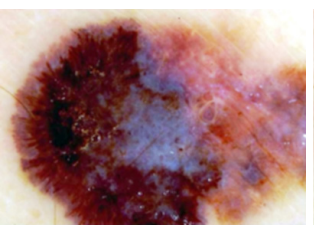

(k)

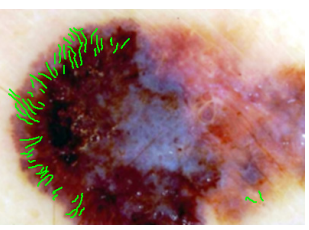

(1)

Fig. 4. Qualitative results. (a), and (c) illustrate absent lesions with their results in (b) and (d). (e) and (g) are starburst lesions. Their regular streaks are shown in (f) and (h). Two melanomas with irregular streaks are shown in the third row, (i) and (k), with their results in (j) and (l).

(called $L C T$ ). $L C T$ includes the following 13 features: The mean, standard deviation and reciprocal of coefficient of variation (mean/stdev) of values in $\mathrm{H}, \mathrm{S}$, and $\mathrm{V}$ from HSV and $\mathrm{L}^{*}$ of $\mathrm{L}^{*} \mathrm{a}^{*} \mathrm{~b}^{*}$, and four of the classical Gray-level co-occurrence matrix based texture measures; energy, contrast, correlation, and homogeneity [4]. Finally, these 25 features are fed into the SimpleLogistic classifier implemented in Weka.

\section{Results}

Using ten-fold cross-validation, we evaluated our proposed approach on streak detection on a set of 300 dermoscopy images, including 105 absent and 195 present. 250 images are chosen randomly from two atlases of dermoscopy [10[1], and 50 images are taken from experts' archives with permission. Corrupted images due to the acquisition parameters such as lighting and magnification, partial lesions (entire lesion was not visible), or lesions occluded with an unreasonable amount of either oil or hair are excluded. Using the L channel of the $L^{*} a^{*} b^{*}$ colour channel as the luminance image for streak detection, we obtained the best performance for both data sets. For 300 images, accuracy of Absent/Present classification using L* is $85 \%$, with AUC of $90.5 \%$. In the second experiment, 200 images (100 starburst, and 100 non-starburst) are used to evaluate the performance of our method on starburst detection. We achieved the accuracy 
Table 1. This table shows results of $L C T$ (first row) and STR (second row) separately and combined (third row) for the multi-scale analysis on the $\mathrm{L}^{*}$ on classifying $N$ lesions with (Present) and without (Absent) streaks. The last row shows the evaluation on another set $(N=200)$ for finding starburst pattern. The last column (AUC) shows the Area Under ROC curve.

\begin{tabular}{|l|l|l|l|l|l|l|l|}
\hline Experiment & $\mathbf{N}$ & Features & Prec. & Recall & FMeasure & Acc. & AUC \\
\hline \hline \multirow{3}{*}{ Abs/Pres } & 300 & LCT & 0.712 & 0.722 & 0.713 & 0.722 & 0.779 \\
\cline { 2 - 7 } & STR & 0.835 & 0.837 & 0.835 & 0.837 & 0.901 \\
\cline { 2 - 7 } & & LCT+STR & $\mathbf{0 . 8 5}$ & $\mathbf{0 . 8 7}$ & $\mathbf{0 . 8 6}$ & $\mathbf{0 . 8 5}$ & $\mathbf{0 . 9 0 5}$ \\
\hline \hline Starburst & 200 & LCT+STR & $\mathbf{0 . 8 1 5}$ & $\mathbf{0 . 8 1 5}$ & $\mathbf{0 . 8 1 5}$ & $\mathbf{0 . 8 1 5}$ & $\mathbf{0 . 8 7 7}$ \\
\hline
\end{tabular}

of $81.5 \%$ and AUC of $87.7 \%$ using 10 -fold cross validation. Table 1 reports the details. Assuming the difficulty level of the images in [6] is similar to those of one of our data sets, our approach achieves an AUC of $90.5 \%$ comparing to $80 \%$ reported in [6].

\section{Conclusion}

We have presented an automatic approach for detection of radially oriented streaks on 300 real dermoscopic images, using techniques based on ridge and valley detection used in fingerprint image recognition. We demonstrated that the proposed approach can detect streaks in dermoscopy images and visualize them. Furthermore, with locating streaks and providing a qualitative analysis, it can be used to highlight suspicious areas for experts diagnosis and for visualization and training purposes.

Acknowledgments. This work was funded by the Canadian NSERC, CIHR-Skin Research Training Center and a grant from the Canadian Health Research Project (CHRP).

\section{References}

1. Soyer, H., Argenziano, G., et al.: Dermoscopy of pigmented skin lesions. An atlas based on the Consensus Net Meeting on Dermoscopy 2000. Edra, Milan (2001)

2. Menzies, S., Ingvar, C., McCarthy, W.: A sensitivity and specificity analysis of the surface microscopy features of invasive melanoma. Melanoma Research 6(1), 55-62 (1996)

3. Sadeghi, M., Razmara, M., Lee, T., Atkins, M.: A novel method for detection of pigment network in dermoscopic images using graphs. Computerized Medical Imaging and Graphics 35(2), 137-143 (2011)

4. Sadeghi, M., Razmara, M., Wighton, P., Lee, T.K., Atkins, M.S.: Modeling the Dermoscopic Structure Pigment Network Using a Clinically Inspired Feature Set. In: Liao, H., Eddie Edwards, P.J., Pan, X., Fan, Y., Yang, G.Z. (eds.) MIAR 2010. LNCS, vol. 6326, pp. 467-474. Springer, Heidelberg (2010)

5. Betta, G., Di Leo, G., et al.: Automated application of the 7-point checklist diagnosis method for skin lesions: Estimation of chromatic and shape parameters. In: Proceedings of the IEEE Instrumentation and Measurement Technology Conference, pp. 1818-1822 (2005) 
6. Mirzaalian, H., Lee, T., Hamarneh, G.: Learning features for streak detection in dermoscopic color images using localized radial flux of principal intensity curvature. In: IEEE Workshop on Mathematical Methods for Biomedical Image Analysis, pp. 97-101 (2012)

7. Wighton, P., Sadeghi, M., Lee, T.K., Atkins, M.S.: A Fully Automatic Random Walker Segmentation for Skin Lesions in a Supervised Setting. In: Yang, G.-Z., Hawkes, D., Rueckert, D., Noble, A., Taylor, C. (eds.) MICCAI 2009, Part II. LNCS, vol. 5762, pp. 1108-1115. Springer, Heidelberg (2009)

8. Kass, M., Witkin, A.: Analyzing oriented patterns. Computer Vision, Graphics, and Image Processing 37(3), 362-385 (1987)

9. Hong, L., Wan, Y., Jain, A.: Fingerprint image enhancement: Algorithm and performance evaluation. IEEE Trans. on Pattern Anal and Machine Intelligence 20, 777-789 (1998)

10. Argenziano, G., Soyer, H., et al.: Interactive Atlas of Dermoscopy (Book and CD-ROM). Edra Medical Publishing and New Media (2000) 